\title{
Intrinsically Safe Robot Arm: Adjustable Static Balancing and Low Power Actuation
}

\author{
Mathijs Vermeulen • Martijn Wisse
}

Accepted: 2 March 2010 / Published online: 20 March 2010

(c) The Author(s) 2010. This article is published with open access at Springerlink.com

\begin{abstract}
We present a design for a manipulator that is intrinsically mechanically safe, i.e. it can not cause pain (let alone damage) to a human being even if the control system has a failure. Based on the pressure pain thresholds for human skin, we derive a pinching safety constraint that limits the actuator torque, and an impact safety constraint that results in a trade-off between mass and velocity. To fulfill all constraints, the manipulator requires a spring balancing system that counteracts gravity in all configurations of the manipulator. This allows the use of extremely low-power DC motors (only $4.5 \mathrm{~W}$ ). Thanks to the torque and speed limitations of these motors the manipulator is indeed intrinsically safe, yet still capable of moving a useful payload of $1.2 \mathrm{~kg}$ over a distance of $0.8 \mathrm{~m}$ in $1.5 \mathrm{~s}$.
\end{abstract}

Keywords Safe robot - Gravity compensation - Gravity balancing $\cdot$ Low power robot $\cdot$ Manipulator

\section{Introduction}

The long anticipated introduction of robots into society is now taking place, exemplified by robot lawn mowers and vacuum cleaners. Although there are currently no manipulators for the general public, developments are well underway $[2,19,39]$. This has caused the current increase in research activity on robot safety. Out of the broad spectrum of safety issues, we focus on physical (mechanical) interaction between robot arms and humans.

M. Vermeulen $\cdot$ M. Wisse $(\bowtie)$

Dept. of Mechanical Engineering, Delft University

of Technology, Mekelweg 2, 2628 CD Delft, The Netherlands

e-mail: m.wisse@tudelft.n
After a surge of publications on safety of industrial robots around 1985 (e.g. [4, 12, 24]), there were only a few pertaining studies in the decades after that (e.g. [6, 22, 40, 41]). Only in the last few years, safe physical robot-human interaction has really become an active field of research again (e.g. [3, 7, 16, 18, 21, 25, 28, 37, 42-44]). Very recently, Haddadin et al. [13-15] have produced various publications with a thorough overview on the subject, including interesting impact experiments. The main conclusions are that there are currently no useful measures or indicators for the safety of robot arms; most existing measures stem from the automobile industry and are useful for discriminating between life-threatening and non-life-threatening situations at velocities much higher than those typically used by robot manipulators. The authors also show that it is highly unlikely that their well-designed light-weight robot arm will cause injury to human beings. Our research differs from theirs in that we set a much stricter limit; our manipulator should not even be able to cause pain, even when there is a complete failure of the control system.

In this paper, we present a design for a robot arm that is mechanically intrinsically safe; even with the worst case control signal, it is physically incapable of causing pain to a human being. We used a simple pain threshold to derive the design requirements (Sect. 2). These requirements lead to the conclusion that weight balancing is a necessity (Sect. 3), so the key feature of our design is a weight balancing system using springs (Sect. 4), based on earlier work in our group [30] extended with an actively adjustable balancing system. The prototype design is shown in Sect. 5, and the results and conclusions follow in Sects. 6 and 7. 


\section{Design Requirements}

The intended application of the manipulator is anywhere in the direct environment of human beings for assistive or collaborative tasks. When we started this research project, we initially aimed at applications in fruit/vegetable harvesting and processing, which led to the following requirements: the manipulator should handle payloads of up to $1.2 \mathrm{~kg}$, and it should be able to move $0.8 \mathrm{~m}$ in $1.5 \mathrm{~s}$ [34]. We believe that these numbers are reasonable for domestic tasks as well. The design space for our robot is bounded by these performance requirements, together with the following requirements for pinching safety and impact safety.

\subsection{Pinching Safety}

In quasi-static contact, the manipulator could cause pain if it is able to exert a pressure on the skin that is above the pain threshold. This is a function of the maximal actuator torque, the contact area, and the configuration of the robot. Haddadin [15] shows how the configuration of the arm can provide a high transmission ratio from actuator torque to outward-pushing force, especially near the fully outstretched configuration. In this paper, we ignore this potential safety hazard (leaving it for future improvements of the prototype).

Tests with Algometers [5] and a literature overview of tests [20] provide Pressure Pain Thresholds (PPT's) for different regions of the body. The lowest reported pain tolerance, a PPT of $150 \mathrm{kPa}$, is located in the temporal areas on the head. This threshold was measured with a low pressure application rate $(13.5 \mathrm{kPa} / \mathrm{s})$, which is relevant for static pressure. To be safe, in our design we will use a maximum contact pressure of $100 \mathrm{kPa}$.

We assume that proper design of the exterior shell of the robot arm should lead to an area of contact of at least $5 \mathrm{~cm}^{2}$. This is a conservative estimate for a shell without sharp edges and with a soft rubber covering. This area, together with the maximum allowable contact pressure of $100 \mathrm{kPa}$, leads to the following design constraint:

$F_{\text {Static } \text { ontact }} \leq 100[\mathrm{kPa}] \cdot 5 \mathrm{~cm}^{2}=50 \mathrm{~N}$

Note that this is the maximum allowable force that the actuators are capable to exert at any contact point. This value will ultimately limit the allowable actuator torques and therefore manipulator acceleration.

\subsection{Impact Safety}

During an impact, ultimately it is the same skin pain threshold that determines if the robot causes pain. Thus, the ultimate measure to take into account is again the contact force

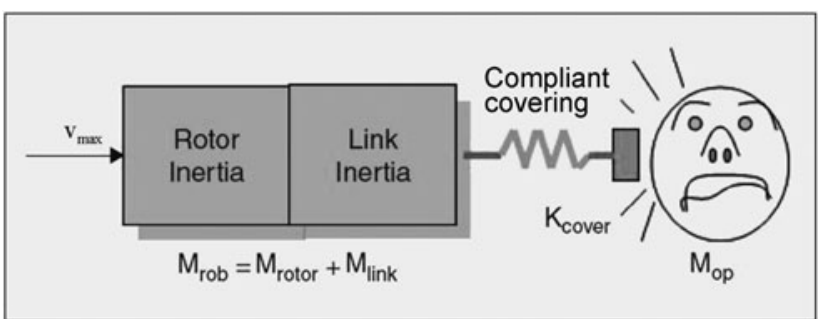

Fig. 1 Simplified model of impact between a one-DOF arm and an operator, figure taken from [3] and adapted

during the impact (and not energy or other impact quantities). A relevant value for the pain pressure threshold at high contact velocities is $P P T=250 \mathrm{kPa}$, as reported by Jensen et al. [20] from experiments with a pressure application rate of $500 \mathrm{kPa} / \mathrm{s}$. Again the temporal area was the most sensitive part of the body.

We use the simple (fully elastic) contact model from Bicchi and Tonietti [3], see Fig. 1, assuming a contact stiffness $k$ of a soft rubber covering of about $5 \mathrm{~N} / \mathrm{mm}$, and again a contact area $A$ of $5 \mathrm{~cm}^{2}$. Note that the mass $m_{r o b}$ represents the effective mass as experienced at the contact point during impact. To calculate the relation between the manipulator velocity and the resulting impact force (at maximal compression of the rubber covering), we use an unconstrained impact model (see [13]) and we model the human head as a rigid object with a mass $m_{o p}$ of $3.5 \mathrm{~kg}$ [10]. This leads to the following design constraint for the robot, which is in fact a trade-off between maximum velocity and effective mass, see also Fig. 2.

$$
\begin{aligned}
v_{\text {max }} & =\frac{A \cdot P P T}{m_{o p}} \cdot \sqrt{\frac{m_{o p}\left(m_{r o b}+m_{o p}\right)}{m_{r o b} k}} \\
& =0.95 \cdot \sqrt{\frac{m_{r o b}+3.5}{m_{r o b}}}
\end{aligned}
$$

For example, this trade-off predicts for a $2 \mathrm{~kg}$ effective mass (which includes the payload and the arm itself) a maximum allowable velocity of $1.57 \mathrm{~m} / \mathrm{s}$.

Note that the velocity is one of the key aspects that should be limited in the mechanical design of our robot if we want it to be intrinsically safe. As remarked by Haddadin [15], it is not necessarily a good idea to use compliant actuation (e.g. proposed by $[3,33,38]$ ), because the potential energy storage in the compliant actuator could lead to velocities that are higher than specified. Therefore, we will use geared DC motors that are directly coupled to the joints.

\section{Need for Static Balancing}

We have calculated that a manipulator should be safe if its weight, velocity and actuator power are limited according 


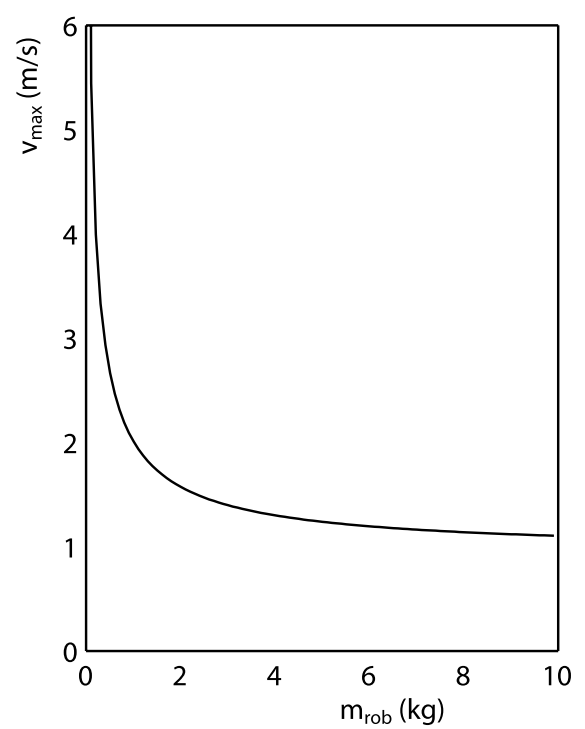

Fig. 2 Impact safety trade-off of $v_{\max }$ versus $m_{r o b}$, with $m_{o p}=3.5 \mathrm{~kg}$ and $k_{\text {cover }}=5 \mathrm{~N} / \mathrm{mm}$, and a maximum dynamic contact pressure of $250 \mathrm{kPa}$. Safe mass-velocity combinations are those below the plotted line

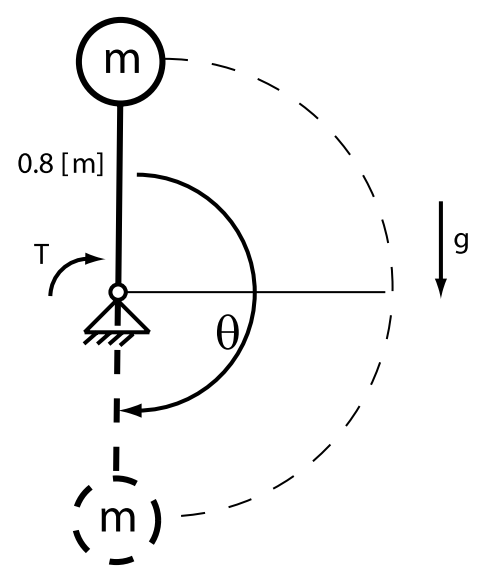

Fig. 3 A $0.8 \mathrm{~m}$ pendulum with a $2 \mathrm{~kg}$ mass attached and a constant torque $T$ falling over an $\pi$ rad angle is calculated to be unsafe

to (1) and (2). Now we want to verify this by constructing and testing a prototype. Here a fundamental problem arises: the torque required for lifting the weight of the object alone will make that the manipulator can easily violate both safety criteria when moving downward. The situation is sketched with a simple one-DOF pendulum in Fig. 3. The static holding torque $T$ is based on the assumption that the actuation should at least be able to hold the pendulum when it is in horizontal position: $T=m g l$.

The mass $m$ is a $2 \mathrm{~kg}$ mass. It represents the payload mass, chosen in Sect. 2, the hand mass and the mass of the arm. Hand mass is not worked out further in this paper, but a mass of $800 \mathrm{~g}$ is reserved for arm and hand, which should be sufficient $[8,23]$. The arm length $l$ is chosen as $0.8 \mathrm{~m}$.
The pinching safety criterion from (1) is violated. The torque $T$ is about $16 \mathrm{Nm}$, and in a worst-case scenario (when the arm is moving downward from a horizontal position), a torque exerted by the mass of $16 \mathrm{Nm}$ is added. The combined torque means an excess of the allowed $50 \mathrm{~N}$ from shoulder up to $640 \mathrm{~mm}$ from the shoulder, or on $80 \%$ of the arms length, which is not allowable.

Also the impact safety criterion from (2) is violated for the situation in Fig. 3. The velocity in downward position of the initially static pendulum is obtained by observing the potential and kinetic energy and added work by $T$ in the system:

$$
\begin{aligned}
& E_{\text {potential }}+W=E_{\text {kinetic }} \\
& m g(l+l)+m g l \theta=\frac{1}{2} m l^{2} \dot{\theta}^{2}
\end{aligned}
$$

Solving (3) results in a velocity of $9.0 \mathrm{~m} / \mathrm{s}$. From Fig. 2 it is clear that an impact with a $2 \mathrm{~kg}$ mass with this velocity will definitely cause pain.

The solution is to make the manipulator statically balanced. In a statically balanced system there is a constant net force equal to and opposing the gravity force and there is no net change in potential energy. Therefore no static holding torque is required, resulting in low required actuator torques.

Although a counterweight could be used for static balancing, we find the added inertia of this solution undesirable. Springs could be used as an alternative. In a well designed spring-balanced system the total potential energy in mass and spring remains constant, independent of the configuration of the manipulator. This concept was generalized for one degree-of-freedom by Streit and Gilmore [29] and later extended to more dimensions for various robots [1, 9, $11,26,27,31,35,36]$. To allow the manipulator to handle a range of payloads, the balancing system will have to be adjustable.

\section{Adjustable Static Balancing}

Static balancing is a straight-forward idea, but it becomes quite complex when multiple degrees of freedom are involved and when adjustability is required. In this section we will first identify the locations and order of the degrees of freedom. Next, we will develop the geometric equations and implement adjustability.

The locations of the joints are chosen in accordance with those of a human arm. This means the manipulator consists of a three-DOF "shoulder" and a one-DOF "elbow". A three-DOF shoulder is necessary for the manipulator to be able to move over low objects and around high objects.

In choosing the order of joints, the occurrence of gimbal lock is an important issue [32]. The shoulder and elbow 
Fig. 4 Gimbal lock can occur in both order of joints. (a) Order $z y x x$ is locked when 2 nd joint is rotated over $\pm 90^{\circ}$, one degree of rational freedom left. (b) Order $z x y x$ is locked when 2nd joint is rotated over $\pm 90^{\circ}$, but two degree of rotational freedom left (a) zyxx order
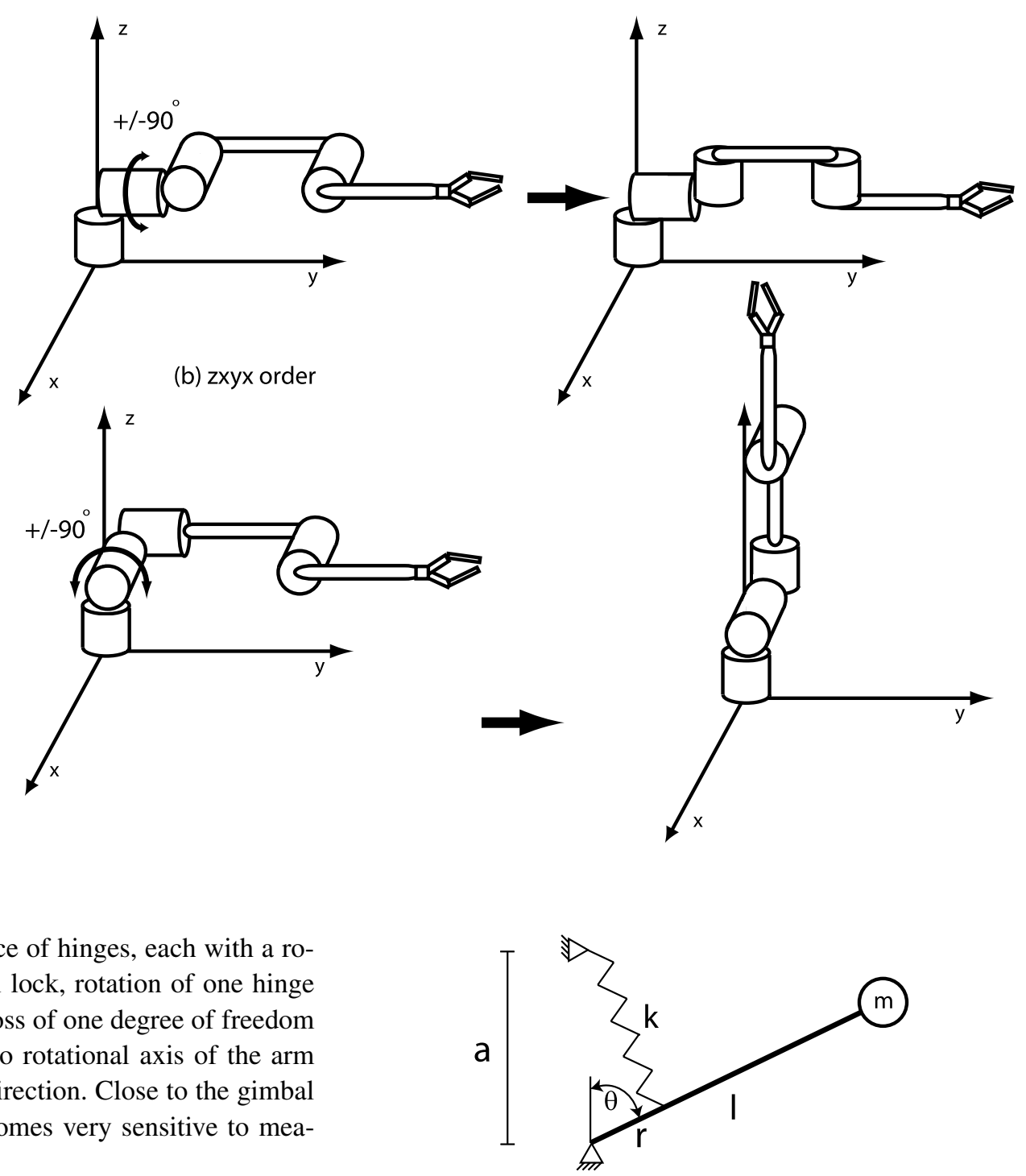

Fig. 5 Basic gravity equilibrator of a balanced mechanism. A lever with length $l$ can freely rotate about one end over angle $\theta$, has a mass $m$ attached to the other end and has an ideal spring with stiffness $k$ attached at distance $r$ from the rotation point

spring's other end is attached to the ground at distance $a$ directly above the joint. 'Ideal' means that the spring is linear and has no zero length. With help of the cosine rule, the total amount of potential energy $\left(V_{t o t}\right)$ in the system can be expressed as:

$V_{\text {tot }}=m g l \cos (\theta)+\frac{1}{2} k\left(a^{2}+r^{2}\right)-a k r \cos (\theta)$

The total energy in (4) remains constant, independent of angle $\theta$, when $m g l=a k r$. The system is in perfect balance.

In [30] a concept for a four-DOF balanced robot arm is presented, with the order of joints we desire. The basic equilibrator is extended to four-DOF with help of the modifi- 
(a)

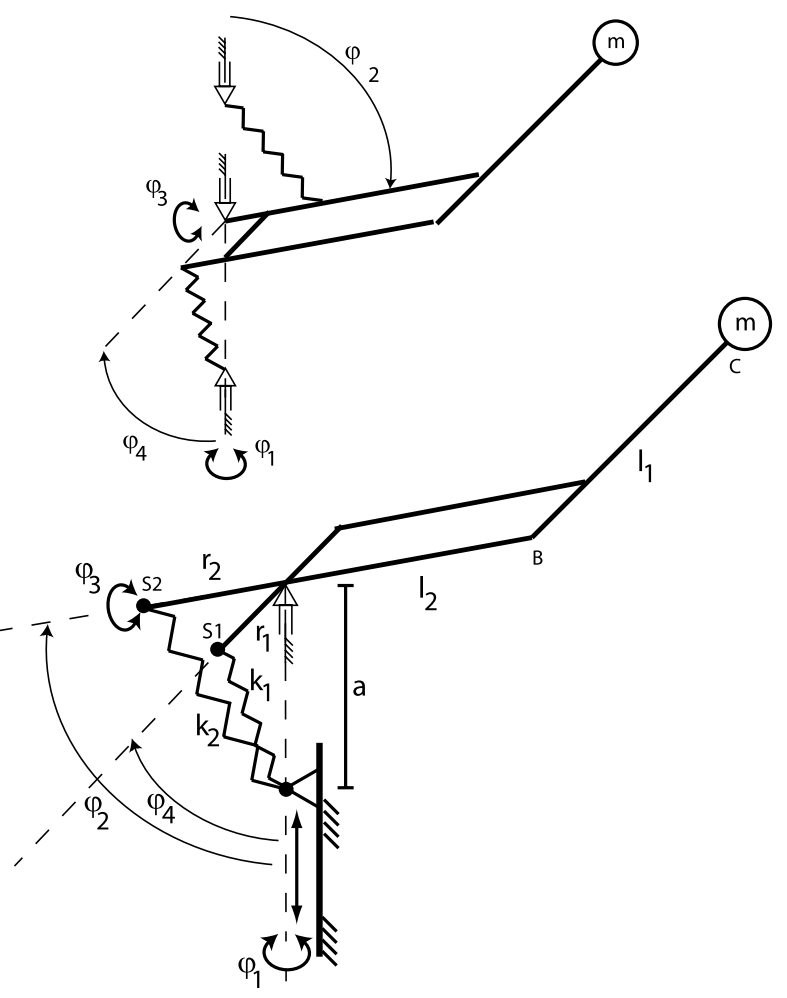

Fig. 6 (a) Four-DOF static balancing concept [30]. (b) Adjustable static balancing concept showing degrees of freedom, dimensions of elements and spring attachment points

cation rules explained by Herder [17]. These rules explain how to add degrees of freedom while keeping a perfectly balanced system. A parallelogram is used to place the lower arm compensating spring above the shoulder, see Fig. 6 a.

The four-DOF concept can be used as a basis for the adjustable static balancing concept, only a number of adjustments are required. First, in the original design the parallelogram is placed below the shoulder, occupying most of the space below the shoulder. The structure supporting the arm would therefore have to be placed above the shoulder, which is not desirable for many applications. Second, the ideal springs are sticking out above and below the shoulder, occupying a lot of space. It would be favorable to place both below the shoulder.

The concept from [30] is modified to the adjustable balancing concept drawn in one plane in Fig. $6 \mathrm{~b}$ by using similar design steps as described in [30]. The parallelogram is moved to above the shoulder and both springs are now located below the shoulder and attached on one end to the same location, which also simplifies the design for adjustability, as will be shown later. The range of $\phi_{3}$ is now restricted to $180^{\circ}$ from the drawn situation in one direction, because otherwise the springs can touch and cross each other. However, this range limitation forms no limitation for the utility of the total arm.
To prove that the new concept is perfectly balanced, the model is parameterized using Euler Angles. Conversion from local to global coordinates is done, following the system of axes from Fig. 4, with $R_{x}, R_{y}$, and $R_{z}$ denoting 3D rotation matrices around the $x, y$, and $z$ axes respectively. Relative $(x, y, z)$ coordinates of elbow $\left(B_{0}\right)$, end effector $\left(C_{0}\right)$ and the spring attachments on the arm, $S 1_{0}$ and $S 2_{0}$, are as follows:

$$
\begin{array}{ll}
B_{0}=\left[\begin{array}{l}
0 \\
l_{2} \\
0
\end{array}\right], & C_{0}=\left[\begin{array}{l}
0 \\
l_{1} \\
0
\end{array}\right], \\
S 1_{0}=\left[\begin{array}{c}
0 \\
-r_{1} \\
0
\end{array}\right], & S 2_{0}=\left[\begin{array}{c}
0 \\
-r_{2} \\
0
\end{array}\right]
\end{array}
$$

The lower spring attachment is located on the $z$-axis through the shoulder at distance $a$ below this joint. Rotation over angle $\phi_{1}$, perpendicular to the direction of gravity, is not relevant here for the balancing equations. The rotation matrices and relative coordinates from (5) are used to obtain expressions for global end effector and spring attachments coordinates:

$$
\begin{aligned}
& C=R_{x}\left(\phi_{2}\right) R_{y}\left(\phi_{3}\right) B_{0}+R_{x}\left(\phi_{2}\right) R_{y}\left(\phi_{3}\right) R_{x}\left(\phi_{4}\right) C_{0} \\
& S 1=R_{x}\left(\phi_{2}\right) R_{y}\left(\phi_{3}\right) R_{x}\left(\phi_{4}\right) S 1_{0}, S 2=R_{x}\left(\phi_{2}\right) S 2_{0}
\end{aligned}
$$

The potential energy of the mass $\left(V_{m}\right)$ and the two springs $\left(V_{e 1}, V_{e 2}\right)$ can be expressed with the coordinates in (6) and (7). The horizontal plane through the shoulder is taken as a reference level for the potential energy of the mass.

$V_{m}=m g h=m g C(3)$

$V_{e 1}=\frac{1}{2} k_{1} \Delta u^{2}=\frac{1}{2} k_{1}\left(S 1(1)^{2}+S 1(2)^{2}+(S 1(3)+a)^{2}\right)$

$V_{e 2}=\frac{1}{2} k_{2} \Delta u^{2}=\frac{1}{2} k_{2}\left(S 2(1)^{2}+S 2(2)^{2}+(S 2(3)+a)^{2}\right)$

The total amount of potential energy in the system is the addition of functions (8), (9) and (10):

$$
\begin{aligned}
V_{\text {tot }}= & \left(m g l_{1}-k_{1} r_{1} a\right) \\
& \times\left(\sin \left(\phi_{2}\right) \cos \left(\phi_{4}\right)+\cos \left(\phi_{2}\right) \cos \left(\phi_{3}\right) \sin \left(\phi_{4}\right)\right) \\
& +\left(m g l_{2}-k_{2} r_{2} a\right) \times \sin \left(\phi_{2}\right) \\
& +\frac{1}{2} k_{2} a^{2}+\frac{1}{2} k_{1} a^{2}+\frac{1}{2} k_{2} r_{2}^{2}+\frac{1}{2} k_{1} r_{1}^{2}
\end{aligned}
$$

The system is in equilibrium, independent of the four angles, when $m g l_{1}=a k_{1} r_{1}$ and $m g l_{2}=a k_{2} r_{2}$. 


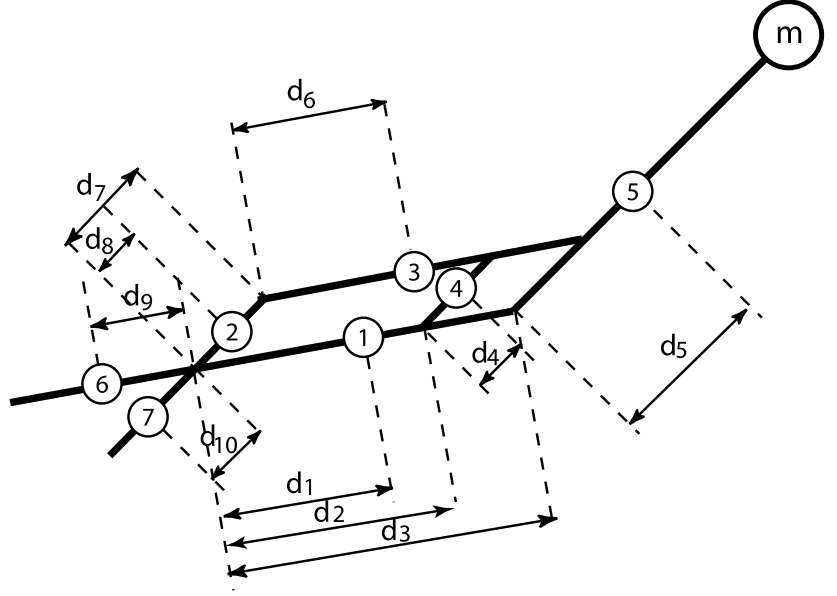

Fig. 7 Masses of elements of the system can be included in the equilibrium equations according to their center-of-mass location

The identified equilibrium equations can be used to make the balancing system adjustable. Mass, gravity and length are properties that are fixed. Adjusting the spring stiffness is possible [17], but solutions are difficult. Adjusting the attachment points of the spring, defined by $a$ and $r$, is the easiest solution. An adjustment system can best be located on the fixed world, in order to keep the inertia of the moving arm as low as possible. Therefore, dimension $a$ is used to recover equilibrium in case of changing mass $m$. One end of the spring attachments could simply be moved upward and downward to adjust $a$, see Fig. $6 b$.

The masses of the links and other system elements are neglected so far, but in the final balancing system design they will have to be taken into account. With the masses of system elements included, both springs are not equally loaded anymore. Therefore, we must distinguish between $a_{1}$ and $a_{2}$. The contribution of the masses in the equilibrium equation is shown in (12) and (13) with dimensions shown in Fig. 7 and is the result of calculations similar to the equilibrium proof.

$\left(m_{1} d_{1}+m_{3} d_{3}+m_{4} d_{9}+m_{5} d_{10}+m_{6} d_{6}+m_{2}\right) g=k_{2} r_{2} a_{2}$

$\left(m_{2} d_{2}+m_{3} d_{8}+m_{4} d_{4}+m_{5} d_{5}+m_{7} d_{7}+m_{1}\right) g=k_{1} r_{1} a_{1}$

\section{Prototype Construction}

Now that the set of requirements and safety criteria have been identified and the adjustable balancing concept has been introduced, the prototype can be designed.

\subsection{Motor Selection}

To select the proper motors for the four degrees of freedom, the desired velocity and the safety constraints have to be taken into account. The required motors are selected by iterating back and forth between a Solidworks ${ }^{\circledR}$ CAD design and dynamical analysis in ADAMS $^{\circledR}$ to monitor the velocity and safety constraints.

The motor selection process of the first and second degree of freedom, $\phi_{1}$ and $\phi_{2}$ in Fig. 6 starts by regarding the manipulator in fully stretched position as a beam rotating around the shoulder joint and identifying the total rotational inertia of this representation. In the CAD system a detailed design for the manipulator, including the static balancing subsystem, is developed. Small aluminum parts and thin-walled steel tubes are used for a fairly lightweight design.

The individual masses of parts of the lower- and upper arm are obtained from this initial design. These masses and their center-of-mass location relative to the shoulder are used in combination with Fig. 7 to get a total rotational inertia of $1.15 \mathrm{~kg} \mathrm{~m}^{2}$.

When the arm is simplified in stretched orientation as a rotating beam, the effective mass on every position on the arm can be calculated by dividing the total inertia by the squared distance $d$ from the shoulder.

$m_{r o b}=\frac{1.15}{d^{2}}$

At the end effector, for example, the effective mass is $1.8 \mathrm{~kg}$. Equations (2) and (14) are combined to form a relation between the maximum velocity on every position on the arm $v_{\max }$ and distance $d$, see also Fig. 8 .

$v_{\max }=0.95 \sqrt{\frac{1.15+3.5 d^{2}}{1.15}}$

The maximum velocity of each motor is determined by looking at the worst case impact situation where a combination of motors is fully actuated. In such a case the massvelocity trade-off for impact safety should not be exceeded on any location on the arm. The highest end effector velocity occurs when the arm is fully stretched, $\phi_{1}, \phi_{2}$ and $\phi_{4}$ are all fully actuated and the resulting axis of rotation of the shoulder is parallel to that of the elbow. The local velocity, expressed in the angular velocities in radians, on every position on the arm, can in that case be expressed as:

$$
v(d)= \begin{cases}\sqrt{\omega_{1}^{2}+\omega_{2}^{2}} \cdot d, & 0<d \leq 0.4 \\ \sqrt{\omega_{1}^{2}+\omega_{2}^{2}} \cdot d+\omega_{4} \cdot d-\omega_{4} \cdot 0.4, & 0.4<d \leq 0.8\end{cases}
$$




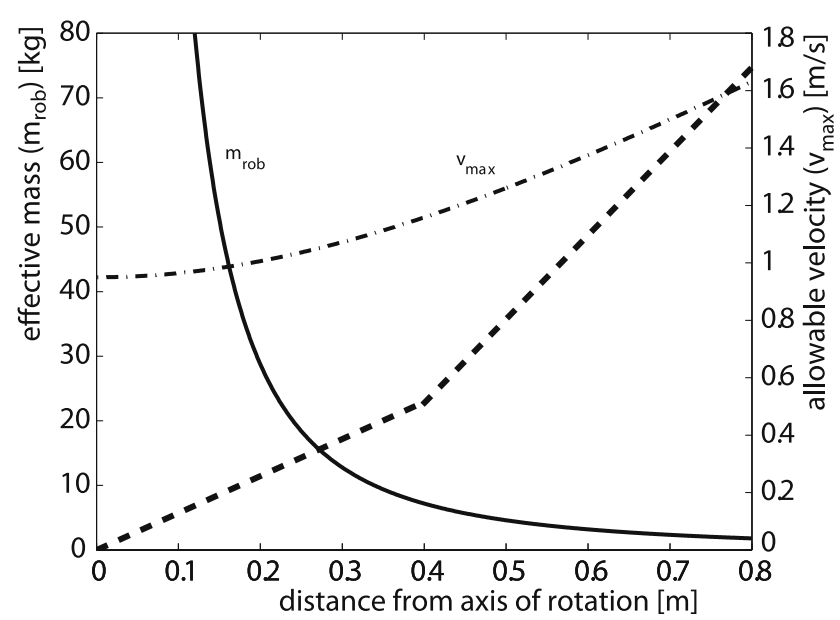

Fig. 8 The effective mass on every position on the arm when it is regarded as a rotating beam, indicated with the black line. The point-dashed line indicates the allowable velocity, following from the impact safety constraint. The velocity resulting from the rotational velocity of the beam should stay under the allowable velocity and is indicated with the dashed line. The bend shows the position where the elbow actuation becomes active, besides the shoulder actuation

Initially, for ease of calculation, the angular velocities are all taken equal. For the maximum allowable end effector velocity from (15) of $1.65 \mathrm{~m} / \mathrm{s}$, the velocity is expressed as:

$\sqrt{2 \cdot \omega^{2}} \cdot 0.8+\omega \cdot 0.8-\omega \cdot 0.4 \leq 1.65$

This results in a maximum allowable angular velocity $\omega$ of $1.1 \mathrm{rad} / \mathrm{s}$. To guarantee absolute intrinsic safety on the level of actuation, the motors should have a no-load-speed at which the manipulator moves slower than (or equal to) the maximum allowable angular velocity. In normal operation, each motor will then operate close to full speed.

For both $\phi_{1}$ and $\phi_{2}$, a $4.5 \mathrm{~W}$ Maxon Motor A-max26 with a 190:1 planetary gearbox and a 2:1 pulley transmission are chosen. For the reduction of the no-load-speed of the motor to the maximum allowable angular velocity, a large gear ratio is required. However, this ratio will also result in a large output torque, running the risk to exceed the pinching safety constraint from Sect. 2.1. To avoid this, a low power motor is chosen. Because the available gearboxes could not handle the required torque, part of the reduction is covered by a pulley transmission with an estimated efficiency of $80 \%$. Including the $70 \%$ efficient gearbox, a maximum speed of about $1.0 \mathrm{rad} / \mathrm{s}$, which is allowable, and a maximum torque of $8.9 \mathrm{Nm}$ result.

Because of limitations by the pinching safety criteria from Sect. 2.1, this torque is just acceptable. Increasing the maximum torque would increase operating velocity, but also the pinching force. At current torque, the force of $50 \mathrm{~N}$ $\left(100 \mathrm{kPa} \cdot 5 \mathrm{~cm}^{2}\right)$ allowed by the criterion is exceeded in worst case at a distance of about $250 \mathrm{~mm}$ from the shoulder. This worst case is when $\phi_{1}$ and $\phi_{2}$ are both actuated

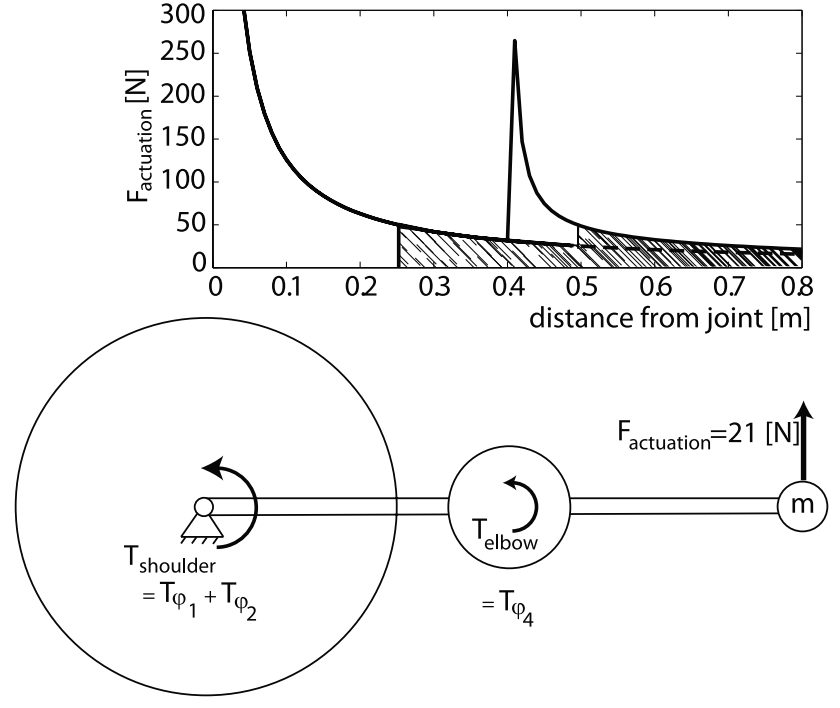

Fig. 9 Actuation force by torques $T_{\text {shoulder }}$ and $T_{\text {elbow }}$ is reduced to pinching safety limits by adding spheres around the joint. Black line indicates the combined force of both actuators, the dashed line is the contribution of the shoulder actuation only. Top of the hatched area indicates the force including the spheres, which always stays below $50 \mathrm{~N}$

at full torque simultaneously. So the complete lower arm is safe and the first $250 \mathrm{~mm}$ of the upper arm must be covered, for example with a sphere around the shoulder, see Fig. 9. A sphere with such a radius is just doable, a higher torque resulting in a larger sphere is unfavorable.

In Adams/View ${ }^{\circledR}$ a model, rotating the arm over $\phi_{1}$, is built that moves the end-effector over a $0.8 \mathrm{~m}$ distance. That is a rotation of the manipulator of $57^{\circ}$ within $1.3 \mathrm{~s}$, which is sufficiently fast. The model uses a symmetrical bangbang control signal, and the motor characteristics (maximum torque, speed/torque gradient, and rotor inertia) are taken into account. After tuning the torque durations, the final position is reached in $1.3 \mathrm{~s}$, see Fig. 10, which is even faster than the required $1.5 \mathrm{~s}$. This leaves room for decreasing velocity when friction, which is not taken into account, has effect in the prototype. Without friction, $\phi_{1}$ and $\phi_{2}$ are equal in the model, so $\phi_{2}$ actuation is also acceptable.

To find the maximum allowable angular velocity $\omega_{4}$, (16), the chosen maximum angular velocities of $\phi_{1}$ and $\phi_{2}$ of $1.0 \mathrm{rad} / \mathrm{s}$ and the maximum allowable end effector velocity of $1.65 \mathrm{~m} / \mathrm{s}$ are combined:

$\sqrt{1.0^{2}+1.0^{2}} \cdot 0.8+\omega_{4} \cdot 0.8-\omega_{4} \cdot 0.4 \leq 1.65$

This results in an allowable angular velocity $\omega_{4}$ of $1.3 \mathrm{rad} / \mathrm{s}$. A $2.5 \mathrm{~W}$ Maxon Motor A-max19 with a 128:1, $59 \%$ efficient, planetary gearbox and a 5:1 pulley coupling with again $80 \%$ expected efficiency is chosen. An allowable maximum speed of $1.4 \mathrm{rad} / \mathrm{s}$ and a maximum torque of $2.34 \mathrm{Nm}$ result. This velocity is slightly higher than acceptable, but since transmission comes in discrete steps and this 


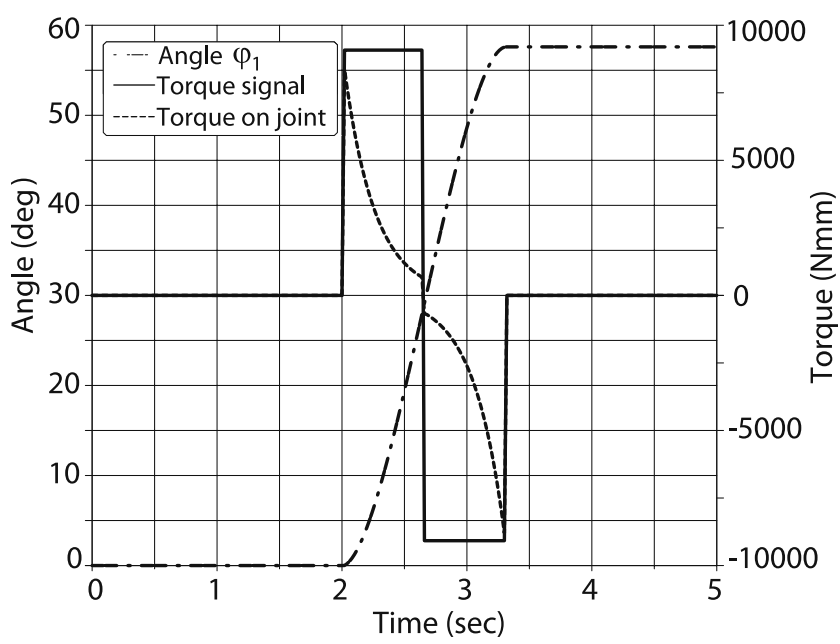

Fig. 10 Adams/View ${ }^{\circledR}$ results for a one-DOF arm model, including constant speed/torque gradient motor behavior. Drawn are the used control signal, resulting torque over $\phi_{4}$ and change of angle. The final position is reached after $1.3 \mathrm{~s}$

transmission gives velocity most nearest to the allowable, we decide to accept it. Moreover, in practice, friction will lower the maximum velocity. The maximum local velocity for all motors of the worst case combined according to (16) is plotted in Fig. 8 and indeed, the maximum allowable velocity is not significantly exceeded on any location.

In a simulation, rotating $\phi_{4}$ over $115^{\circ}$, the trajectory of $0.8 \mathrm{~m}$ is finished after $1.4 \mathrm{~s}$. The maximum pinching force in the worst case explained in the maximum velocity calculations is exceeded at $95 \mathrm{~mm}$ from the elbow, requiring a sphere with this radius, see Fig. 9.

Rotation over $\phi_{3}$ is not used in any worst case situation. For ease of calculation, the same motor and gear combination as used for $\phi_{4}$ is used for $\phi_{3}$.

\subsection{Spring Compensation System}

For the calculation of the dimensions of the spring compensation system and the springs to use, (12) and (13) are used in an iterative process between the CAD model and available springs from catalogs.

The masses of the components of the arm and the relative positions of their center of mass from the CAD model are filled in the equations, resulting in the torque equations. These are $k_{2} r_{2} a_{2 \max }=9.38 \mathrm{Nm}$ and $k_{1} r_{1} a_{1 \text { max }}=8.48 \mathrm{Nm}$, including the maximum payload, and $k_{2} r_{2} a_{2 \min }=4.67 \mathrm{Nm}$ and $k_{1} r_{1} a_{1 \min }=3.77 \mathrm{Nm}$, with an empty hand. Because of reasons of implementation, we take $k_{1}=k_{2}$ and $r_{1}=r_{2}$.

To make the balancing compensation adjustable, the length of $a$ from Fig. 6 must be moved between a minimum and a maximum value.

For the desired range of payload mass, $a_{1 \text { min }}$ and $a_{2 \text { min }}$ should be able to increase with a factor 2.5 from the small- est value $\left(\left(m_{\text {payload }}+m_{\text {hand }}\right) / m_{\text {hand }}\right)$, so a too high minimum $a$ value would blow up the system size. However, because of limited space in the design around the shoulder joint, both $a_{1}$ and $a_{2}$ also have a low bound of $90 \mathrm{~mm}$. This minimum value is chosen for $a_{1 \min }$. Because of the difference in mass of both equations, $a_{2 \min }$ should always be slightly larger. But $a_{2 m i n}$ is always a fixed distance larger than $a_{1}$ and therefore both springs could still be connected to a single translating mechanism. The fixed distance is about $21 \mathrm{~mm}\left(\frac{4.67-3.77}{3.77} \cdot 90 \mathrm{~mm}\right)$, resulting in a minimum $a_{2 \mathrm{~min}}$ value of $111 \mathrm{~mm}$.

Distance $r$ should be as high as possible to avoid high forces in the elements and joints around the shoulder. However, $r$ cannot exceed $a$, to avoid contact between the two attachment points. A difference of $6 \mathrm{~mm}$ leaves enough room in both spring systems, resulting in an $r$ value of $84 \mathrm{~mm}$. Combining all information in the equations results in a stiffness $k$ value of $500 \mathrm{~N} / \mathrm{m}$.

$a_{1 \max }$ and $a_{2 \max }$ are respectively $202 \mathrm{~mm}$ and $223 \mathrm{~mm}$, based on the torque equations and the values for $k$ and $r$. This means that both $a_{1}$ and $a_{2}$ are maximally increased by $112 \mathrm{~mm}$.

In the final design with the identified dimensions of the spring compensation system, the arm range, defined according to Fig. 6, is limited to $[-0.5 \pi, 0.5 \pi] \mathrm{rad}$ for $\phi_{1}$, $[0.16 \pi, 0.83 \pi] \mathrm{rad}$ for $\phi_{2},[-0.6 \pi, 0] \mathrm{rad}$ with respect to vertical for $\phi_{3}$ and $[-0.5 \pi, 0.83 \pi] \mathrm{rad}$ for $\phi_{4}$. This results in a maximum ideal spring length of about $242 \mathrm{~mm}$ for spring 1 and $194 \mathrm{~mm}$ for spring 2. Using springs with preload simulate an ideal (zero length at zero force) spring [17] is not possible, because this cannot be produced. Therefore, cable pulleys are required to simulate ideal springs [17].

The extension stroke of the available springs with a stiffness of about $500 \mathrm{~N} / \mathrm{m}$ is about $179 \mathrm{~mm}$, which is not sufficient. A spring stroke amplifier with an amplification of 2:1 provides the desired stroke, but also demands 4 times the spring stiffness. This stiffness-stroke combination is also not available. The solution is to use two parallel springs with respective stiffness values of $1210 \mathrm{~N} / \mathrm{m}$ and $520 \mathrm{~N} / \mathrm{m}$ and stroke $171 \mathrm{~mm}$ and $222 \mathrm{~mm}$. This provides the desired stiffness and stroke. The spring with lowest stiffness fits entirely inside the high stiffness spring and both springs are of equal length, so the parallel springs can easily be used in the design.

The design of the spring stroke amplification subsystem is drawn in Fig. 11. The springs are attached between the set of pulleys and the lower attachments and the subsystem is completely moved up and down to adjust $a$. The attachment point of the cable for spring 2 on the mechanism is moving in a plane and stays always left from the subsystem as shown in Fig. 11. The point of the cable for spring 1, however, is not constrained to a plane and can be left and right from the subsystem. Therefore, a bar passively rotating over angle $\Psi$ 


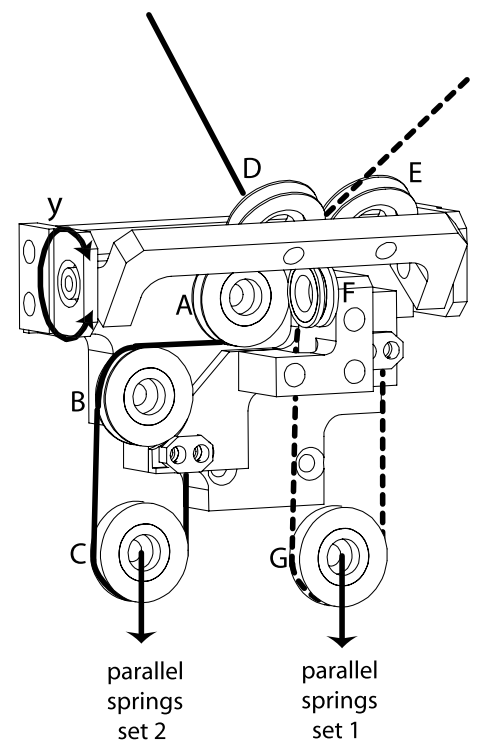

Fig. 11 Spring stroke amplifier subsystem. Parallel springs set 2 is connected by a cable guided through pulleys A, B and C. Parallel springs set 1 is connected by a cable though pulleys D or E, depending on the arms orientation, and F and G. D and $\mathrm{E}$ are connected to a bar rotating passively over angle $\Psi$ to keep the pulleys in line with the cable

is included to make sure the pulleys stay in line with the cable and when the cable switches from left to right, pulley (E) takes over from (D). Finally, pulley (B) makes sure that the springs cannot touch each other.

\subsection{Final Design}

With the actuation selected and the spring compensation system designed, the final design, drawn in Fig. 12 and shown in Fig. 13, concludes the design phase.

Both springs and the spring stroke amplification subsystem are attached to a T-profile (A) that moves up and down along a linear slide mechanism. The profile is long enough to include the length of the fully stretched springs $(300 \mathrm{~mm})$. The mechanism consists of a rail with two carriages at a fixed distance, of which the upper one is driven by an internal spindle mechanism with pitch $0.1 \mathrm{inch}$. The spindle is driven by a Maxon RE-35 DC motor with a 66:1 gearbox. The rail is long enough to house the T-profile and to move it over the desired $112 \mathrm{~mm}$ distance. Note that this actuator was not yet incorporated in the safety considerations and was not actuated during the experiments in this paper; in future research we intend to calculate the maximum allowable speed of the balancing adjustment actuator.

The linear slide mechanism is attached to a housing that is rotated over an angle $\phi_{1}$ by a Maxon A-max 26 actuation combination. This housing is connected to the rest of the manipulator, so the balancing system is rotating along with the arm. The manipulator is connected to the fixed world by ball bearings above and below the linear slide.

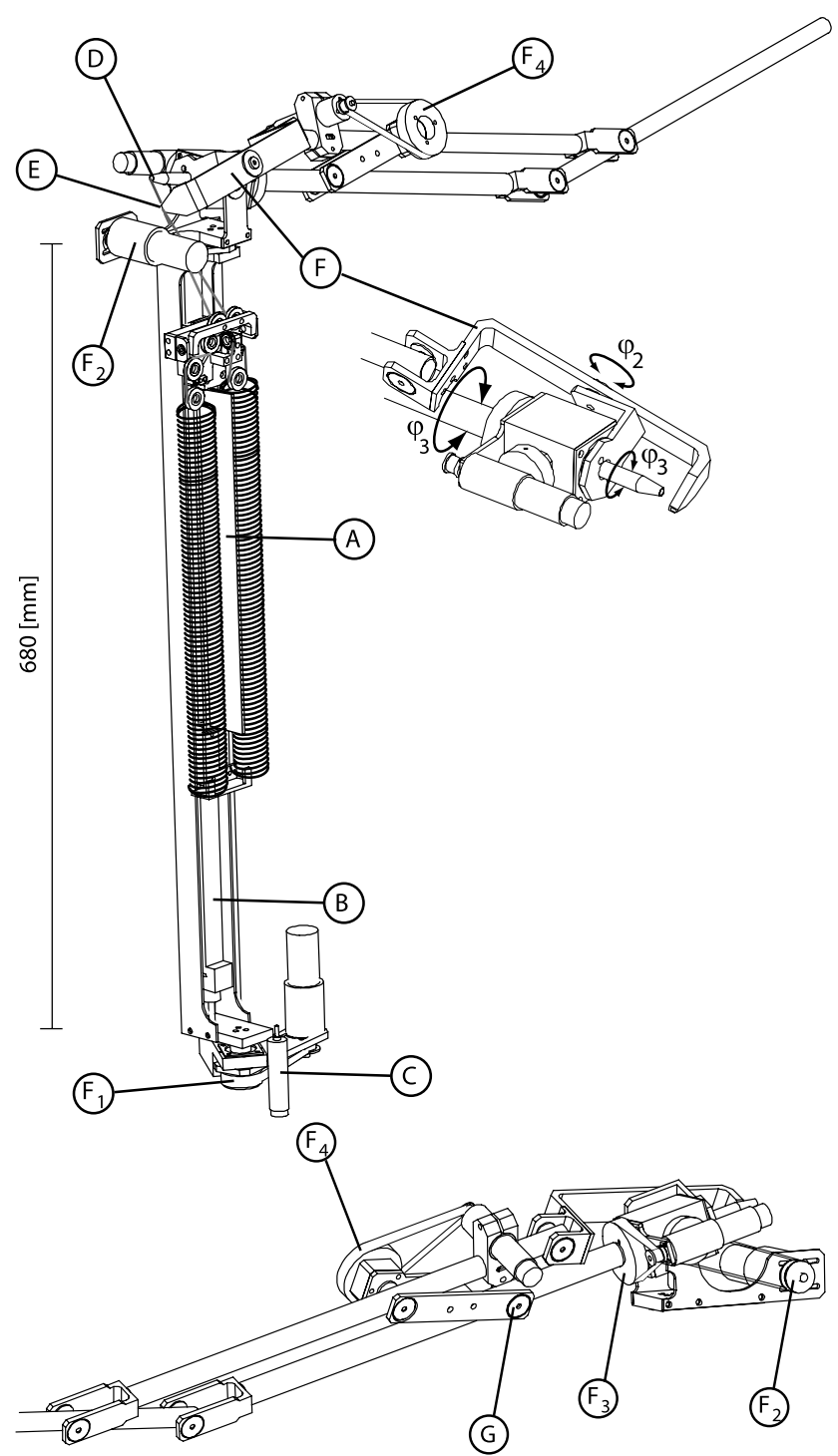

Fig. 12 Complete design of the manipulator (above) and part of the manipulator viewed from the other side (below) Actuation subsystems, including motors, pulleys and belts, of the 1st, 2nd, 3rd and 4th degree of freedom (resp. $\phi_{1}, \phi_{2}, \phi_{3}$ and $\phi_{4}$ ). Springs and spring stroke amplifiers (A) are moved up and down by a linear slide (B), actuated by a motor (C). Cables are attached at shoulder (D) and at the end (E) of the rotating bar subsystem $(\mathrm{F})$, see inserted detail. For all joints roll bearings are used $(\mathrm{G})$

The cable from spring 2 is connected at attachment (D), that moves in a plane rotating along with the manipulator. The attachment is located on a prolongation of the upper arm through the shoulder joint.

For the attachment of the cable from spring 1 (E) however, no more room directly through the shoulder joint is available. To solve this, a rotating bar subsystem (F) is used, see inserted detail in Fig. 12. It diverts the forces around the shoulder joint, while allowing rotations over $\phi_{2}$ and $\phi_{3}$ as if the mechanism goes directly through the joint. 


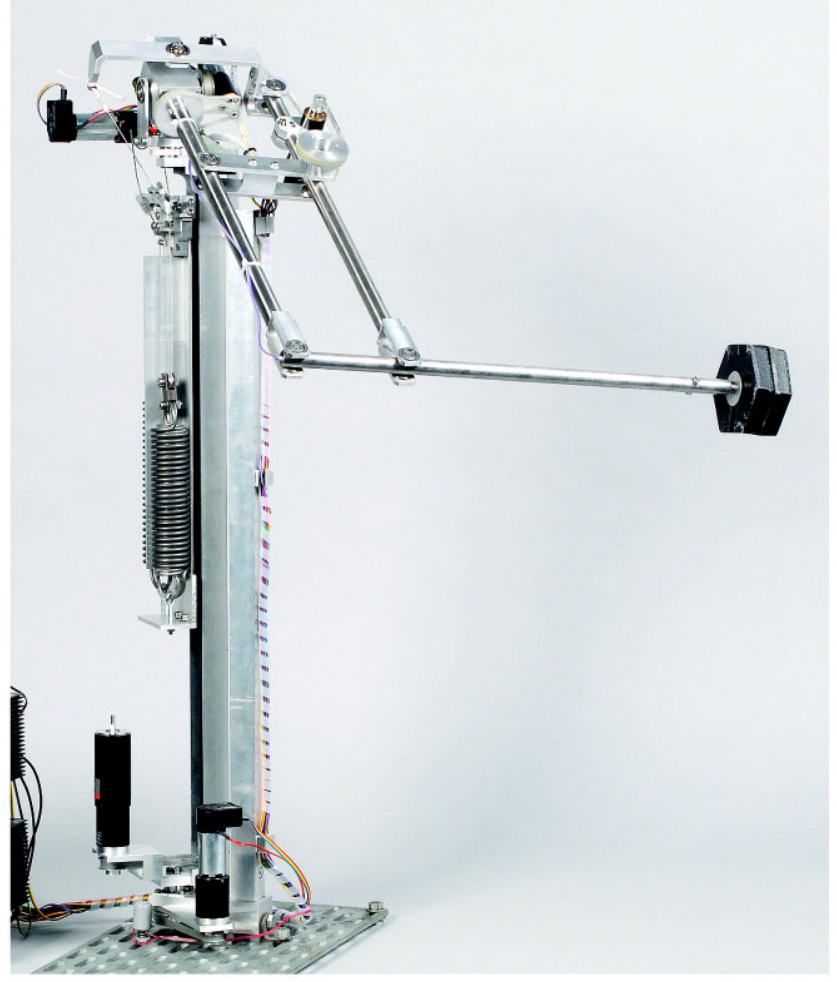

Fig. 13 Manipulator prototype

For active rotation over $\phi_{3}$ and $\phi_{4}$ a Maxon A-max 19 actuation combination is used. Active rotation over $\phi_{2}$ is done by a Maxon A-max 26 actuation combination. In all five actuation combinations, adjustable motor positions are used to bring the belts under tension.

\subsection{Equipment, interface and software}

For controlling the manipulator a PC/104 computer with the Matlab Simulink xPCtarget environment is used. A 16 bit IO card is used for switch readouts and for analog output to the four linear amplifiers that drive the motors. A 16 bit incremental encoder card is used for motor encoder readout.

\section{Experiments and Results}

\subsection{Is the Manipulator Industrially Applicable?}

To see if the manipulator can move with the desired velocity, first three primary movement operations used in pick and place activities are identified, see Fig. 14. For each of these operations the manipulator should be able to move the payload over the desired trajectory of $0.8 \mathrm{~m}$ in $1.5 \mathrm{~s}$. Movement over $\phi_{3}$ is not regarded as a primary operation in picking and placing and can be done more slowly. All experiments were performed with a $2 \mathrm{~kg}$ mass attached to the end of the manipulator. (a)
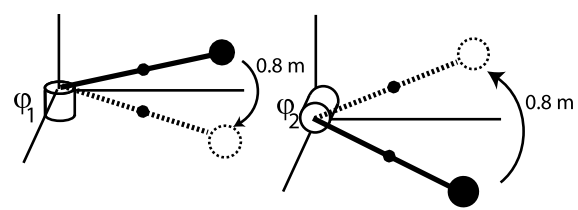

(b)

(c)

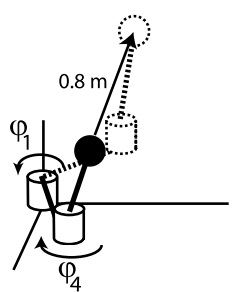

Fig. 14 Three primary movement operations, where the end effector is moved over a $0.8[\mathrm{~m}]$ distance

(a)

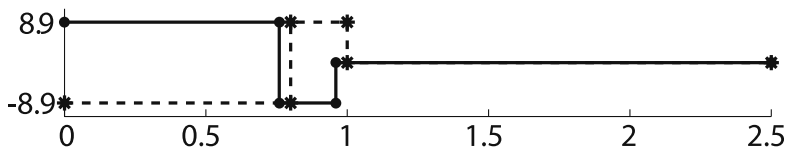

(b)

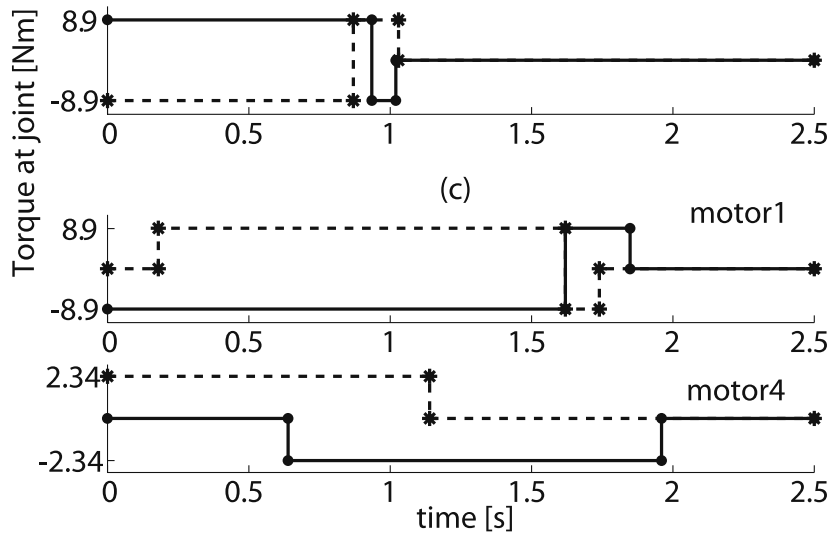

Fig. 15 Open loop control torques used in the velocity tests with the three primary movements. For (a) and (b) positive and negative movements are respectively indicated with a thick and a dashed line. For test (c) control of motor 1 and motor 4 are shown in the upper and lower diagram. Stretching and compacting are respectively indicated with a thick and a dashed line. The open loop control signals were manually tuned so that a desired motion was obtained despite significant friction effects

Open loop (manually tuned) bang-bang control is used as it provides the fastest way to go from one location to another. Please note that closed-loop control is not considered in this paper, as we aim to make the system mechanically intrinsically safe for any control signal. For each of the primary movements both the acceleration- and the deceleration phase are performed with maximum control torque. The duration of each phase is adjusted manually, see Fig. 15, to arrive and remain at the desired final position, see Fig. 16. Each primary movement is performed in the direction indicated in Fig. 14, but also in reversed direction to check if results are direction dependent. After each test the data is logged and the arm is manually put back in starting position.

All movements show a non-symmetrical control torque pattern, in contrast with the perfect symmetrical pattern 
Fig. 16 Experimental results of the industrial applicability tests. Shown are primary movements (a) and (b) in positive (thick line) and negative (dashed line) direction and movement (c) in stretching (thick line) and compacting (dashed line) movement. The plots show 'bends' due to the elasticity of the arm construction. A better controller design would improve this behavior, but that is outside the scope of this paper

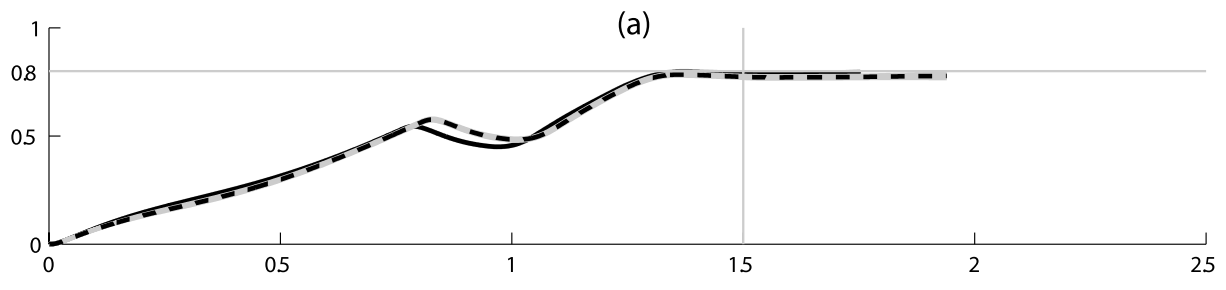

(b)
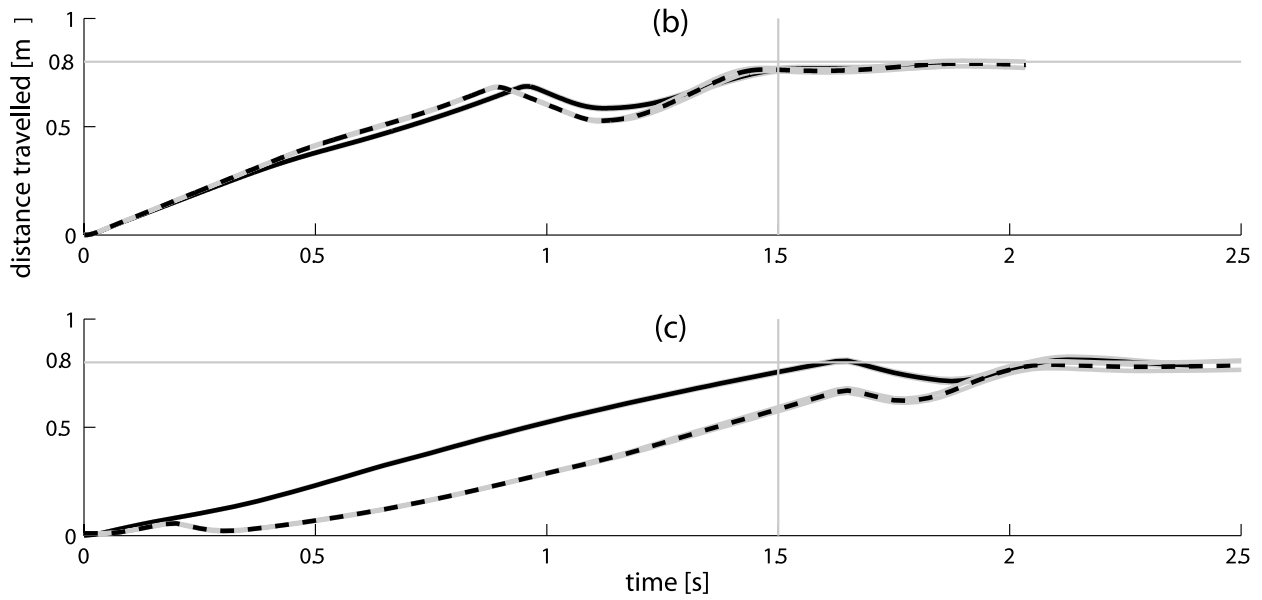

from Fig. 10. This was to be expected, as friction, which plays a significant role, was not taken into account in the simulation. They also have a slightly different control torque pattern for both directions. Possible reasons for this are direction dependent friction in the joint or a slight offset in control system or amplifiers.

The positions plotted in Fig. 16 all show a bend at initiation of deceleration. Reasons for this are the elasticity of the arm structure and the fact that the end effector position is estimated with motor encoder information. Thus, although we set out to design an actuation system without compliance, it turns out that the drive belts still introduce significant compliance.

The final position for both directions of primary movement (a) is reached after about $1.3 \mathrm{~s}$, which is sufficiently fast. Primary movement (b) suffers more from friction than primary movement (a) as it is also connected to the spring compensation system. Therefore it requires more torque to accelerate and less torque to decelerate. It is also slower and arrives at the desired distance in about $1.5 \mathrm{~s}$, just fast enough. For primary motion (c) two motors need to be controlled, but to make a $0.8 \mathrm{~m}$ displacement, both must not be controlled simultaneously. More difficult manual adjustments were required. We decided to adjust both torque profiles to let the end effector move more or less in a straight line. Perhaps another trajectory would have been faster, but this goes beyond the scope of this article. The end effector reaches the final position in about $2.1 \mathrm{~s}$, which is not fast enough.

Although primary movement (c) is not sufficiently fast, we believe that the prototype setup is capable of performing all three primary movements within the desired time. Further research is required to optimize trajectory control.

\subsection{Is the Manipulator Safe?}

Whether the manipulator is safe depends on whether it meets the pinching and impact safety constraints defined in Sect. 2.

The pinching safety constraint of $50 \mathrm{~N}$ was checked by measuring the maximum torques of actuation subsystems $\phi_{1}, \phi_{2}$ and $\phi_{4}$ with a force dynamometer. Measured torques were respectively 7.8, 7.9 and $2.7 \mathrm{Nm}$. This means torques of subsystems $\phi_{1}$ and $\phi_{2}$ are respectively $12 \%$ and $11 \%$ lower than in simulation and the torque of $\phi_{4}$ is $15 \%$ higher than in simulation. These differences can be explained by inaccurate motor catalogue values (friction and efficiency values are easily off by 10\%) and by the fact that we used an assumed value for the efficiency of the coupling between the pulleys.

Pinching safety is guaranteed on the upper arm from $220 \mathrm{~mm}$ from the shoulder and on the lower arm from $100 \mathrm{~mm}$ from the elbow. To calculate this, the same worst case approach as in Sect. 5 and Fig. 9 is used, this time with measured torques. These results show that the manipulator requires an elbow protection sphere with a slightly larger radius for the manipulator to be safe.

To see if the impact safety constraint is met, not the impact safety trade-off should be used, because it is based on an assumed model. Instead, the underlying allowable peak acceleration should be used. 

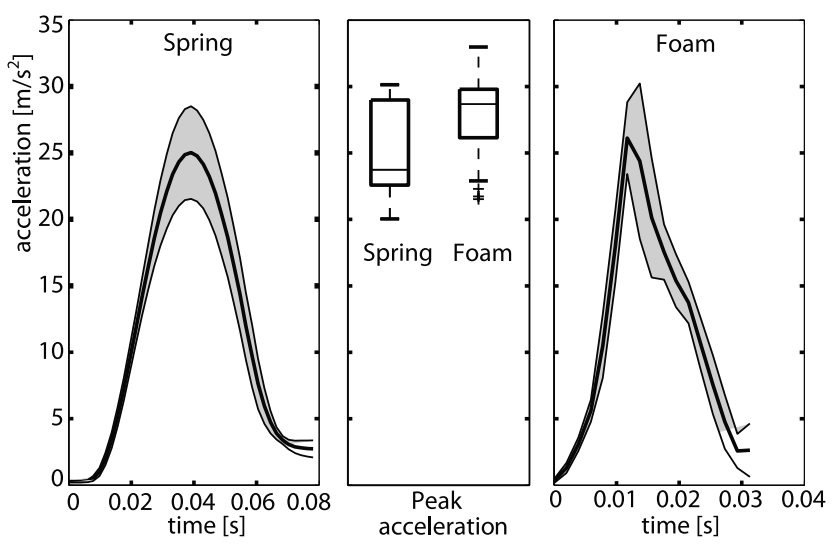

Fig. 17 Measured acceleration during collision of manipulator, rotating over $\phi_{1}$, with human head substitute. The mean and standard deviation values are shown, to the left for collision with a spring and to the right for collision with a $1 \mathrm{~cm}$ thick foam cover in between. Shown in the middle are a boxplots of the measured peak acceleration values. Maximum acceleration peaks measured for spring and foam are respectively 30.5 and $33.1 \mathrm{~m} / \mathrm{s}^{2}$

Safety was checked by measuring the peak acceleration of a $3.5 \mathrm{~kg}$ human head substitute after collision with the manipulator. Measurements were done with an Xsens inertial sensor and logged at $500 \mathrm{~Hz}$, which is the maximum. The manipulator was moving at maximum velocity and with the maximum $2 \mathrm{~kg}$ payload attached. From the test for industrial applicability, primary movement (a) proved to be the fastest and it also has the highest maximum velocity, with velocities up to $0.94 \mathrm{~m} / \mathrm{s}$. Therefore, this rotation was used for all impact safety experiments.

First, experiments with a compression spring in between head and manipulator were performed to check the model results. Unfortunately, only a spring with a slightly higher stiffness of $5.5 \mathrm{~N} / \mathrm{mm}$ was available. Because elasticity in the manipulators mechanism can cause the maximum end effector velocity to slightly deviate, the starting position of the manipulator was varied between about 350 and $450 \mathrm{~mm}$ from the point of collision, the region with the highest velocities. Actuation was stopped manually just after collision, when the head came loose of the manipulator again. The 16 measured collisions resulted in a standard deviation of about $3.5 \mathrm{~m} / \mathrm{s}^{2}$ at peak acceleration measured, see Fig. 17. The highest peak acceleration measured was $30.5 \mathrm{~m} / \mathrm{s}^{2}$ and the maximum contact forces is in that case $107 \mathrm{~N}$. This force is acceptable; according to Sect. 2.2 it has to stay below $250 \mathrm{kPa}$ at an area of $5 \mathrm{~cm}^{2}$, i.e. below $125 \mathrm{~N}$.

Final experiments were performed with foam rubber in between. Foam rubber was chosen as a permanent covering of the manipulator. Different types of foam were considered. Foam types with relatively low damping turned out not to be suitable, because neither of them could deliver the desired $5.5 \mathrm{~N} / \mathrm{mm}$ stiffness for the chosen area of contact of $5 \mathrm{~cm}^{2}$ and at the same time provide the stroke needed. Dur- ing small tests they were compressed entirely and caused the manipulator and the head to endure full impact, resulting in extremely high accelerations. Increasing foam thickness would increase the stroke, but also decrease the stiffness.

As an alternative, tests with Otto Bock VE-RL foam with high damping were performed, using the same method as the spring experiment. At first attempt, a $10 \mathrm{~mm}$ thick foam block with a $5 \mathrm{~cm}^{2}$ area of contact was used. Results of 22 tests show a peak acceleration of $33.1 \mathrm{~m} / \mathrm{s}^{2}$, which means a $116 \mathrm{~N}$ contact force, see Fig. 17. This force is just acceptable (just below $125 \mathrm{~N}$ ) and we decide not to further decrease the foams thickness and use the current thickness.

From the plot it is clear, that the acceleration peak duration is much shorter for foam. Since acceleration is of the same order, this means less energy is absorbed by the head. The most probable explanation is that the highly damping foam absorbs most energy. The shorter duration in combination with the limited sample frequency of $500 \mathrm{~Hz}$ makes that the plot is not looking smooth.

\section{Conclusions}

In this paper, we present a design for an intrinsically mechanically safe manipulator that cannot cause pain to human beings even in the event of catastrophic control failure. The manipulator can move a payload of $1.2 \mathrm{~kg}$ over a distance of $0.8 \mathrm{~m}$ in $1.5 \mathrm{~s}$. The pinching safety constraint prescribed that contact forces should remain below $50 \mathrm{~N}$, which affects the maximal acceleration of the manipulator. The impact safety constraint provided a trade-off between the velocity and the effective mass of the manipulator. Together, these constraints necessitate the use of weight balancing using springs, such that low torque and low power actuators can be used because they need not deliver work against gravity.

Experiments show that the desired operating velocity can be reached for two of the three primary movements. For the third primary movement improved trajectory control is expected to improve operating velocity up to the desired velocity. Pinching safety is guaranteed for about the same areas of the arm as predicted in the design phase, from $220 \mathrm{~mm}$ from the shoulder up to the elbow and from $100 \mathrm{~mm}$ from the elbow up to the end effector. Impact safety of the manipulator moving at maximum velocity, with maximum payload and with a foam rubber cover is proven in collision experiments by measuring the peak acceleration.

From the experiments we concluded that a statically balanced manipulator and payload, with an effective end effector mass of $2 \mathrm{~kg}$, designed by following the safety constraints, can move with sufficient velocity, while guaranteeing intrinsic safety defined by the safety constraints. 
Acknowledgements We would like to thank Just Herder for his advise and Jan van Frankenhuyzen and John Dukker for their help to realize the prototype.

Open Access This article is distributed under the terms of the Creative Commons Attribution Noncommercial License which permits any noncommercial use, distribution, and reproduction in any medium, provided the original author(s) and source are credited.

\section{References}

1. Agrawal SK, Fattah A (2004) Gravity-balancing of spatial robotic manipulators. Mech Mach Theory 39(12):1331-1344

2. Albu-Schäffer A, Haddadin S, Ott C, Stemmer A, Wimböck T, Hirzinger G (2007) The dlr lightweight robot: design and control concepts for robots in human environments. Ind Rob Int $\mathbf{J}$ 34(5):376-385

3. Bicchi A, Tonietti G (2004) Fast and soft-arm tactics. IEEE Robot Autom Mag 11(2):22-33

4. Bonney MC, Yong YF (eds) (1985) Robot safety. IFS Publications/Springer, Berlin

5. Buchanan HM, Midgley JA (1987) Evaluation of pain threshold using a simple pressure algometer. Clin Rheumat 6(4):510-517

6. Corke PI (1999) Safety of advanced robots in human environments: a discussion paper. Proc IARP

7. De Luca A, Albu-Schäffer A, Haddadin S, Hirzinger G (2006) Collision detection and safe reaction with the dlr-iii lightweight manipulator arm. In: IEEE-RSJ international conference on intelligent robots and systems, pp 1623-1630

8. Dechev N, Cleghorn WL, Naumann S (2001) Multiple finger, passive adaptive grasp prosthetic hand. Mech Mach Theory 36(10):1157-1173

9. Fattah A, Agrawal SK (2006) Gravity-balancing of classes of industrial robots. In: Proc of IEEE international conference on robotics and automation, pp 2872-2877

10. Gekhman D (2006) The mass of a human head. http:// hypertextbook.com/facts/2006/DmitriyGekhman.shtml (accessed March 9, 2010)

11. Gosselin CM (2008) Gravity compensation, static balancing and dynamic balancing of parallel mechanisms. Springer, London, pp 27-48. ISBN 978-1-84800-146-6 (Print), 978-1-84800-147-3 (Online)

12. Graham JH, Meagher JF, Derby SJ (1986) A safety and collision avoidance system for industrial robots. IEEE Trans Ind App IA22(1):195-203

13. Haddadin S, Albu-Schäffer A, Hirzinger G (2008) The role of the robot mass and velocity in physical human-robot interactionpart I: Non-constrained blunt impacts. In: Proc of IEEE international conference on robotics and automation, pp 1339-1345

14. Haddadin S, Albu-Schäffer A, Hirzinger G (2008) The role of the robot mass and velocity in physical human-robot interactionpart II: Constrained blunt impacts. In: Proc of IEEE international conference on robotics and automation

15. Haddadin S, Albu-Schäffer A, Hirzinger G (2009) Requirements for safe robots: measurements, analysis and new insights. Int J Robot Res. doi: $10.1177 / 0278364909343970$

16. Heinzmann J, Zelinsky A (2003) Quantitative safety guarantees for physical human-robot interaction. Int J Robot Res 22(78):479-504

17. Herder JL (2001) Energy-free systems: theory, conception and design of statically balanced spring mechanisms. PhD thesis, Delf University of Technology

18. Ikuta K, Ishii H, Nokata M (2003) Safety evaluation method of design and control for human-care robots. Int $\mathrm{J}$ Robot Res 22(5):281-298
19. Jain A, Kemp CC (2009) El-e: an assistive mobile manipulator that autonomously fetches objects from flat surfaces. Auton Robots. doi:10.1007/s10514-009-9148-5

20. Jensen K, Andersen HO, Olesen J, Lindblom U (1986) Pressurepain threshold in human temporal region: evaluation of a new pressure algometer. Pain 25(3):313-323

21. Kulic D, Croft E (2007) Pre-collision strategies for human robot interaction. Auton Robots 22(2):149-164

22. Lim H-O, Tanie K (2000) Human safety mechanisms of humanfriendly robots: passive viscoelastic trunk and passively movable base. Int J Robot Res 19(4):307-335

23. Matsuoka Y (1997) The mechanisms in a humanoid robot hand. Auton Robots 4(2):199-209

24. Nagamachi M (1986) Human factors of industrial robots and robot safety management in Japan. Appl Ergonom 17(1):9-18

25. Oberer S, Schraft RD (2007) Robot-dummy crash tests for robot safety assessment. In: Proc of IEEE international conference on robotics and automation, pp 2934-2939

26. Rahman T, Ramanathan R, Seliktar R, Harwin W (1995) A simple technique to passively gravity-balance articulated mechanisms. J Mech Des 117:655-658

27. Segla S, Kalker-Kalkman CM, Schwab AL (1998) Statical balancing of a robot mechanism with the aid of a genetic algorithm. Mech Mach Theory 33:163-174

28. Shin D, Sardellitti I, Khatib O (2008) Hybrid actuation approach for human-friendly robot design. In: Proc of IEEE international conference on robotics and automation

29. Streit DA, Bj Gilmore (1989) Perfect spring equilibrators for rotatable bodies. J Mech Transm Autom Des 111(12):451-458

30. Tuijthof GJM, Herder JL (2000) Design, actuation and control of an anthropomorphic robot arm. Mech Mach Theory 35(7):945962

31. Ulrich N, Kumar V (1991) Passive mechanical gravity compensation for robot manipulators. In: Proc of IEEE international conference on robotics and automation, pp 1536-1541

32. van der Helm FCT (1997) A standardized protocol for motion recordings of the shoulder. In: First conference of the international shoulder group, pp 7-12

33. Vanderborght B, Verrelst B, Ham RV, Damme MV, Lefeber D, Duran B, Beyl P (2006) Exploiting natural dynamics to reduce energy consumption by controlling the compliance of soft actuators. Int $\mathbf{J}$ Robot Res 25(4):343-358

34. Vermeulen MMA, Wisse M (2008) Maximum allowable manipulator mass based on cycle time, impact safety and pinching safety. Ind Rob Int J 35(5):410-420

35. Walsh GJ, Streit DA, Gilmore BJ (1991) Spatial spring equilibrator theory. Mech Mach Theory 26(2):155-170

36. Wang J, Gosselin CM (2000) Static balancing of spatial fourdegree-of-freedom parallel mechanisms. Mech Mach Theory 35:563-592

37. Wassink M, Stramigioli S (2007) Towards a novel safety norm for domestic robots. In: Proc of IEEE-RSJ international conference on intelligent robots and systems, pp 3354-3359

38. Wolf S, Hirzinger G (2008) A new variable stiffness design: matching requirements of the next robot generation. In: Proc of IEEE international conference on robotics and automation, pp 1741-1746

39. Wyrobek KA, Berger EH, Van der Loos HFM, Salisbury JK (2008) Towards a personal robotics development platform: Rationale and design of an intrinsically safe personal robot. In: Proc of IEEE international conference on robotics and automation, pp 2165-2170

40. Yamada Y, Hirasawa Y, Huand S, Umetani Y (1996) Fail-safe hu$\mathrm{man} /$ robot contact in the safety space. In: IEEE international workshop on robot and human communication, pp 59-64 
41. Yamada Y, Suita K, Imai K, Ikeda H, Sugimoto N (1996) A failure-to-safety robot system for human-robot coexistence. Robot Auton Syst 18:283-291

42. Zinn M, Khatib O, Roth B (2004) A new actuation approach for human friendly robot design. Int J Robot Res 23:379-398

43. Zinn M, Khatib O, Roth B, Salisbury JK (2002) Towards a human-centered intrinsically safe robotic manipulator. In: IARPIEEE/RAS joint workshop on technical challenges for dependable robots in human environments, Toulouse, France

44. Zurada J, Wright AL, Graham JH (2001) A neuro-fuzzy approach for robot system safety. IEEE Trans Syst Man Cybern, Part C, Appl Rev 31(1):49-64
Mathijs Vermeulen received his M.Sc. degree in Mechanical Engineering from Delft University of Technology, The Netherlands, in 2008. He is currently active as a consultant for large technical development projects.

Martijn Wisse received his M.Sc. and Ph.D. degrees in Mechanical Engineering from Delft University of Technology, The Netherlands, in 2000 and 2004, respectively. He is currently active as an associate professor at Delft University of Technology in the field of Humanoid Robotics. 